\title{
Impact of the Hydrometeor Vertical Advection Method on HWRF's Simulated Hurricane Structure
}

\author{
SHAOWU BAO \\ Coastal Carolina University, Conway, South Carolina \\ L. BERNARDET \\ Developmental Testbed Center, and Global Systems Division, National Oceanic and Atmospheric \\ Administration/Earth System Research Laboratory, Boulder, Colorado \\ G. THOMPSON \\ National Center for Atmospheric Research, Boulder, Colorado \\ E. KALINA \\ Cooperative Institute for Research in Environmental Sciences, University of Colorado Boulder, and \\ Developmental Testbed Center, and Global Systems Division, National Oceanic and Atmospheric \\ Administration/Earth System Research Laboratory, Boulder, Colorado \\ K. NewMAN AND M. Biswas \\ Developmental Testbed Center, and National Center for Atmospheric Research, Boulder, Colorado
}

(Manuscript received 16 January 2019, in final form 29 January 2020)

\begin{abstract}
The impact of different hydrometeor advection schemes on TC structure and intensity forecasts is examined through the evaluation of HWRF's simulation of tropical cyclones using the operational Ferrier-Aligo (FA) microphysics that uses total condensate advection versus the same scheme but with separate hydrometeor advection (FA-adv). Results showed that FA-adv simulated larger storms. Idealized simulations revealed that the cause of the simulation differences is the characteristics of the vertical profile of cloud water (Qc), which has a sharp gradient near $850 \mathrm{hPa}$, and rainwater (Qr), which is vertically uniform below the melting layer. In FA, the resultant total condensate profile has a gradient near $850 \mathrm{hPa}$ that is smaller than that of Qc but larger than that of Qr. In FA when the total condensate is advected and partitioned back to Qc and Qr, the advection of Qc is underestimated and that of Qr is overestimated than that in FA-adv. The separate advection of hydrometeors in the FA-adv scheme corrected this problem and caused the difference in microphysics and dynamics fields between the two schemes. The greater vertical advection of Qc in FA-adv represents a continual source of extra diabatic heating that leads to a greater integrated kinetic energy (IKE) in the storm simulated by FA-adv than FA. However, the radial distribution of the azimuthally averaged additional diabatic heating in FA-adv caused a sea level pressure adjustment that leads to a weaker maximum wind speed. The warming in the outer rainbands strengthens wind away from the inner core, which causes the simulated storm size to increase.
\end{abstract}

Denotes content that is immediately available upon publication as open access.

Corresponding author: Shaowu Bao, sbao@coastal.edu

\section{Introduction}

Advection is a computationally time-consuming routine in numerical weather prediction (NWP) models (Sobhani and Vento 2017). Therefore, in time-sensitive operational model forecasting, hydrometeors are sometimes summed up before they are advected, so that the model only needs to calculate the advection of the total 
condensate, instead of advecting multiple hydrometeor species. After the advection routine, the total condensate is partitioned back into the individual species using their respective fractions, which are assumed to remain unchanged during the advection process. This strategy is adopted in the Hurricane Weather Research and Forecast (HWRF) system (Biswas et al. 2018) and in the outer domain of the North American Mesoscale (NAM) model (Janjić 2003; Rogers et al. 2009). However, the impacts of such a time-saving technique on the accuracy of the tropical cyclone forecast have not been systematically evaluated.

Such an evaluation might provide direction for potential upgrades for future operational implementations. HWRF's forecast skill has been improving steadily in recent years. Each year, upgrade candidates, such as innovations in physics schemes, data assimilation techniques, and model resolution, are tested using a multiyear sample of cases by the NOAA Environmental Modeling Center (EMC) and the Developmental Test bed Center (DTC). These test and evaluation (T\&E) studies usually include the candidates' track and intensity forecast skill statistics that are used to determine which candidates will be implemented in the next operational upgrade.

Cloud microphysics is an important component in these T\&E activities. A microphysics scheme describes the processes that control the formation of cloud droplets and ice crystals, their growth, and fallout as precipitation. These processes also control the release of latent heat during phase changes. Cloud microphysics comprises a set of processes that modulate the thermodynamical structure and energy distribution of tropical cyclones. NWP model forecasts are sensitive to the choice of microphysics scheme, which is a large source of forecast uncertainty. For hurricane forecasts, it has been demonstrated that microphysics schemes can impact the hurricane track (Fovell and $\mathrm{Su}$ 2007) and intensity (Pattnaik and Krishnamurti 2007; Zhu and Zhang 2006).

Currently, the microphysics scheme used in the operational HWRF is the Ferrier-Aligo (FA) scheme (Aligo et al. 2014, 2018; Biswas et al. 2018). It is a modified version of the original Ferrier scheme used in NCEP's mesoscale models (Ferrier et al. 2002; Rogers et al. 2001) with an enhanced representation of storm structure over the CONUS and simulations of the continental distribution of hydrometeors (especially ice concentration) and precipitation fall speeds that are more realistic than its predecessors.

$T \& E$ activities were conducted in HWRF with the goal of substituting the single, combined total condensate species with the more typical configuration that advects individual species of hydrometeors (FA-adv)
(Lin et al. 1983; Morrison and Milbrandt 2015; Ferrier 1994; Thompson and Eidhammer 2014). The impact of advecting the total condensate has not previously been evaluated, and the complex HWRF modeling system has been tuned and optimized with such inherent uncertainty of advecting total condensate versus individual hydrometeor species. Therefore, comparing the HWRF simulated hurricane structure and intensity using the total condensate advection against results obtained with separate hydrometeor species advection could shed light on this uncertainty and help represent microphysical processes more realistically in HWRF.

With the release of HWRF 3.9a (Biswas et al. 2017), either of the two choices became available to the research community: one with separate hydrometeor species advection and the other with the total condensate advection. This option provided an excellent opportunity to study how the simulated hurricane structure and intensity differ due to the different advection schemes used.

In this paper we address the impact of using the total condensate advection by evaluating and diagnosing HWRF's FA and FA-adv microphysics schemes using GOES-13 brightness temperatures. Detailed numerical case studies, as well as idealized sensitivity experiments, are used to obtain a better understanding of how the two advection methods affect cloud structure and tropical cyclone characteristics, with the goal of providing insight for HWRF's future improvement.

The paper is organized as follows. Section 2 describes the HWRF model, observational data, cases studied, and evaluation methods. Forecast results are given in section 3, discussions and idealized sensitivity experiments in section 4 , followed by the conclusions in section 5 .

\section{Model and methods}

HWRF v3.9a was used in this study. This version corresponds to the 2017 operational HWRF and employs the WRF v3.9.1 release. In HWRF v3.9a, FA is the default microphysics scheme, but the FA-adv scheme is also available, making it possible to run both the FA and FA-adv and make comparisons.

Figure 1 shows the flowchart of the microphysical processes implemented in FA. While only a single total condensed (liquid/solid) hydrometeor species is explicitly advected and predicted, the scheme is internally designed to predict multiple hydrometeor species, including cloud water $(\mathrm{Qc})$, rainwater $(\mathrm{Qr})$, and nonprecipitating (cloud ice) and precipitating ice (snow, graupel, and sleet) denoted by Qi. Internal variables are used to diagnose the fraction of all liquid in the form of 


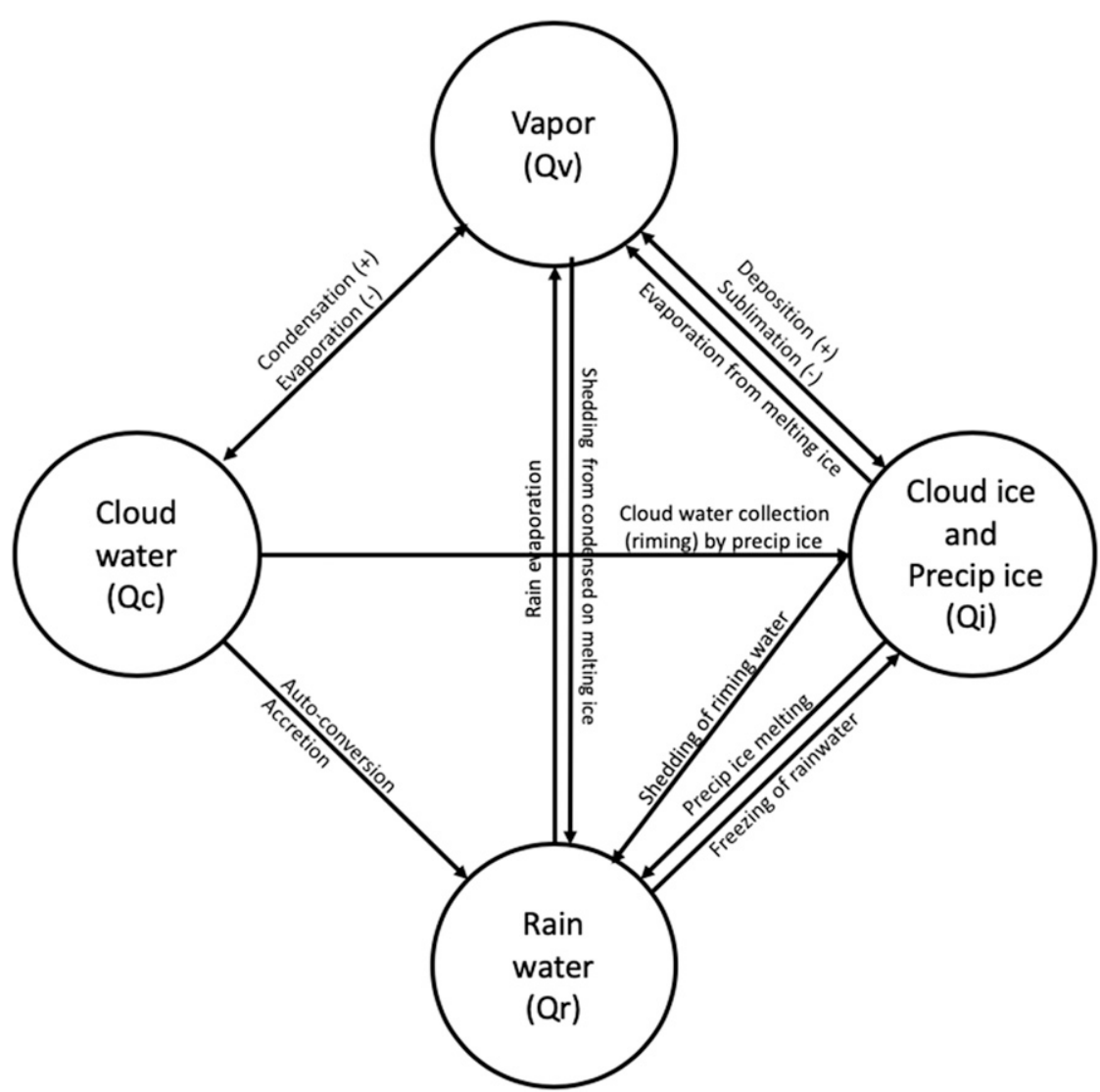

FIG. 1. Flowchart of diagram of the microphysical processes in the Ferrier-Aligo scheme.

rain $\left(F_{r}\right)$ as well as partitioning between snow and graupel using a riming factor. Water vapor changes into Qc by condensation when the moist air is carried by the updraft to the lifting condensation level and reaches saturation. Conversely when air becomes subsaturated and Qc exists, evaporation occurs. During condensation, latent heat is released and warms the air, a main energy source for TC development. Qc can further change into rainwater through auto-conversion and rain accretion, and into ice through freezing and/or collection by precipitation ice. Latent heat is obviously considered with phase changes such as freezing, melting, deposition, and sublimation. Note that the FA scheme in HWRF has only the total condensate advection, as described above.

The simulated cases include Hurricanes 1) Hermine (09L) 29 August 2016-2 September 2016, 2) Matthew (14L) 29 September 2016-9 October 2016, and 3) Jimena (13E) 28 August 2016-7 September 2016. These are all recent TCs that have been tested extensively by EMC and DTC. In this study, these three hurricanes were simulated using the HWRF in NOAA's high-performance computation facility, emulating the HWRF's operational forecast environment as the same configuration and input data were used. As in operations, HWRF simulations were cycled (warm start) except for the first case in each storm. For each simulation, synthetic satellite IR brightness temperature images were generated using the Unified Post Processor (UPP). UPP incorporates the Community Radiative Transfer Model (CRTM) to compute model-derived brightness temperatures for various instruments and channels including IR at the $10.3-\mu \mathrm{m}$ wavelength.

The model-derived synthetic IR brightness temperatures, from simulations using FA and FA-adv, were compared with observations from GOES-13. The observed brightness temperature data have been obtained from the NOAA Comprehensive Large Array-Data Stewardship System (CLASS; www.class.noaa.gov) and regridded to match the model simulation domains. Cold IR brightness temperature areas often correspond to convective cloud tops, while warm areas indicate noncloud or low-altitude clouds. This fact makes IR brightness temperature a relatively simple proxy to evaluate the size and structure of the convection and clouds and to assess the expansive upper-level cold clouds that reside near the tropopause caused by deep convection, such as those that occur in tropical cyclones. Therefore, in addition to the usual TC track and intensity 
comparisons, we will use model synthetic satellite IR brightness temperature to compare against observations, as done by Otkin et al. (2017) and Cintineo et al. (2014).

In addition to the visual comparison of forecast and observed brightness temperature, statistical metrics, such as the probability density function (PDF), were used in the evaluation. The PDF analysis provides information about the fractional amount of high clouds (with colder cloud-top temperature), low clouds (with less cold cloud-top temperature) and nonclouds (warm land or ocean surface). The advantage of these nonlocal PDF metrics is that, since the locations of the grid points are not considered when constructing the PDF, forecasts are not penalized due to tropical cyclone track errors, and the results reflect solely the errors in the simulated hurricane sizes and structures.

To complement the real-data case studies, an idealized configuration of HWRF also employed by Bao et al. (2012), Gopalakrishnan et al. (2011, 2013), and Zhang and Marks (2014) was used to investigate the behavior of the FA and FA-adv schemes. The initial conditions for the idealized HWRF simulations were specified using an idealized prescribed vortex, with a maximum tangential wind speed of $20 \mathrm{~m} \mathrm{~s}^{-1}$ and a radius of maximum wind (RMW) of $90 \mathrm{~km}$, superimposed on a base-state quiescent sounding on an $f$ plane at the latitude of $12.5^{\circ} \mathrm{N}$. The sea surface temperature $(302 \mathrm{~K})$ was time invariant and horizontally homogeneous. No land was present in the simulation domains. These idealized environmental and initial conditions allowed us to isolate the processes to be studied in the absence of the greater complexity of the real case simulations. Although the real-case TC simulations can be compared with observations and performance skill metrics, they can be difficult to analyze due to the complicated highly nonlinear interactions of the environmental fields and the physical processes in the vortex. In this study, the idealized HWRF allowed us to concentrate on the microphysics and advection processes. All the features of the operational version of HWRF, such as the triply nested domain configuration with horizontal grid spacings of 18 , 6 , and $2 \mathrm{~km}$, the vortex following nested grids, and all operational atmospheric physics, were present in the idealized simulations. The UPP was also used to postprocess the idealized HWRF simulation output to obtain the synthetic IR brightness temperature satellite images. Two idealized HWRF simulations with full physics options, one using the FA and the other using the FA-adv microphysics scheme, were run to $126 \mathrm{~h}$, and their simulated hydrometeors, their vertical advection, latent heat release, intensities and storm sizes were compared between FA-adv and FA. To clarify terminology, latent heat release associated with all phase changes of water is referred to as a diabatic process in this paper, although sometimes "moist adiabatic" is used to refer to air parcel motions that involve latent heat release.

\section{Evaluation of results}

The most distinct difference between the FA and FAadv results was the simulated storm size. The synthetic satellite IR brightness temperature images (Fig. 2), with the deep blue indicating the cold cloud tops and the redbrown the warm surface with no-cloud or low-cloud conditions, demonstrates that the simulations with FA and FA-adv predicted similar storm center locations and shapes relative to each other and to the observations. However, in FA-adv, the cloud coverage was noticeably larger than in the FA result, indicating FA-adv may have too much cloud cover. While Fig. 2 only shows a snapshot of one storm, this result is representative of the entire sample over all simulated storms (i.e., FA-adv simulated larger cloud coverage and therefore largersized storms than FA).

Examination of the PDF of brightness temperature also showed larger coverage of cold cloud tops in FAadv than in FA. In Fig. 3, which is from the storm-scale domain that covers an area about $2500 \mathrm{~km} \times 2500 \mathrm{~km}$, the FA-adv showed a larger fraction of cold cloud (180$210 \mathrm{~K}$ ) than the FA and observations. In contrast, the warmest temperatures $(280-300 \mathrm{~K})$ in the FA and FAadv PDF were slightly higher than the observed one. However, on the warm end of the spectrum $(280-300 \mathrm{~K})$ the FA results had a far greater fraction than either observations or the FA-adv results. One caveat to consider in this analysis is that the presence of high clouds in any of the datasets naturally implies the obscuration of any clouds at lower altitudes, so if cloud coverage in general is approximately the same, a simple trade-off will always exist between various temperature ranges, meaning a gain of coverage by one temperature range will lead to the loss by another. In the intermediate range of temperatures $(230-280 \mathrm{~K})$, there is a general lack of sufficient clouds as compared to observations. This phenomenon is quite commonly found in numerical weather prediction models including WRF (Cintineo et al. 2014; Thompson et al. 2016) and the German weather model Consortium for Small-Scale Modeling (COSMO; Eikenberg et al. 2015).

In the literature, TC sizes are often measured as the extent of the $34-\mathrm{kt}\left(1 \mathrm{kt} \approx 0.51 \mathrm{~m} \mathrm{~s}^{-1}\right)$ wind at $10 \mathrm{~m}$ above sea level (AR34) and the radius of the outermost closed sea surface pressure isobar (ROCI) (Carrasco et al. 2014). The difference in TC size between FA and FA-adv results was evident not only in the synthetic IR brightness 

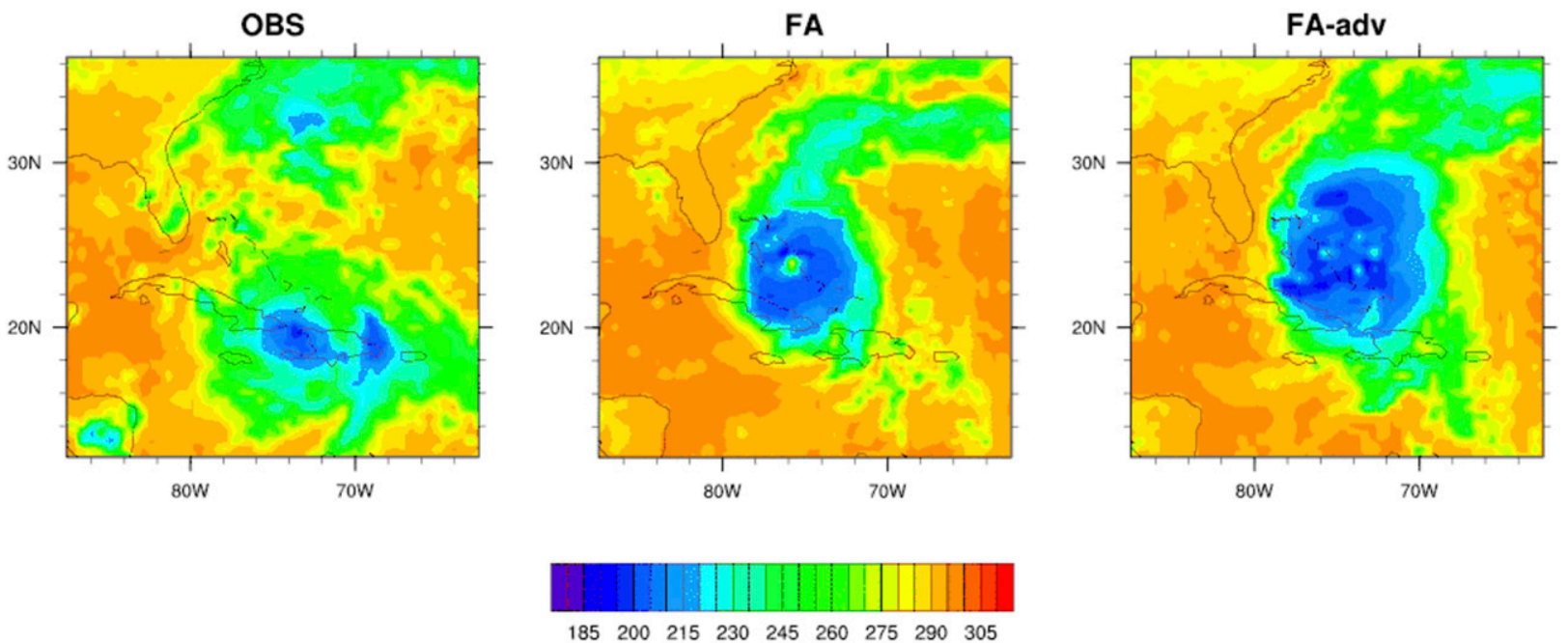

FIG. 2. Snapshot of IR brightness temperature (K) of Hurricane Matthew at the 96-h forecast in the cycle that started at 0000 UTC 1 Oct 2016 on the storm-scale domain that covers about $2500 \mathrm{~km} \times 2500 \mathrm{~km}$. (a) Observed, (b) FA, and (c) FA-adv.

temperature images but also in AR34 and ROCI. Figure 4 shows larger AR34 and ROCI in the forecasts that used the FA-adv. The maximum 10-m wind, however, was about $10 \mathrm{~m} \mathrm{~s}^{-1}$ weaker in FA-adv than in FA in the individual forecast snapshot. That is, in FA-adv the simulated TC was larger but weaker, using the peak $10-\mathrm{m}$ wind as the intensity metric. In Figs. $4 \mathrm{a}$ and $4 \mathrm{~b}$, although the minimum sea level pressure (SLP) in FA-adv was lower than in FA, the SLP contours exhibited a larger gradient in FA than in FA-adv, which will be revisited in section 4 in the discussion of the intensity difference between FA and FA-adv. Figure 4e through Fig. 4h shows that the difference in simulated TC sizes between FA and FA-adv exists not only in individual forecast snapshots but also in a composite storm created by averaging 1763 6-hourly forecast snapshots of Hurricane Matthew, indicating that the pattern applies generally across all the Hurricane Matthew (2016) forecast lead times.

The tendency for weaker storms in FA-adv is also evident in the scatterplot of the pressure-wind $(P-V)$ relationship for all cycles of the Hurricane Matthew cases simulated using FA and FA-adv and in the best track database (Fig. 5). In Fig. 5, the FA-adv results, compared to those from FA, had some $P-V$ points with
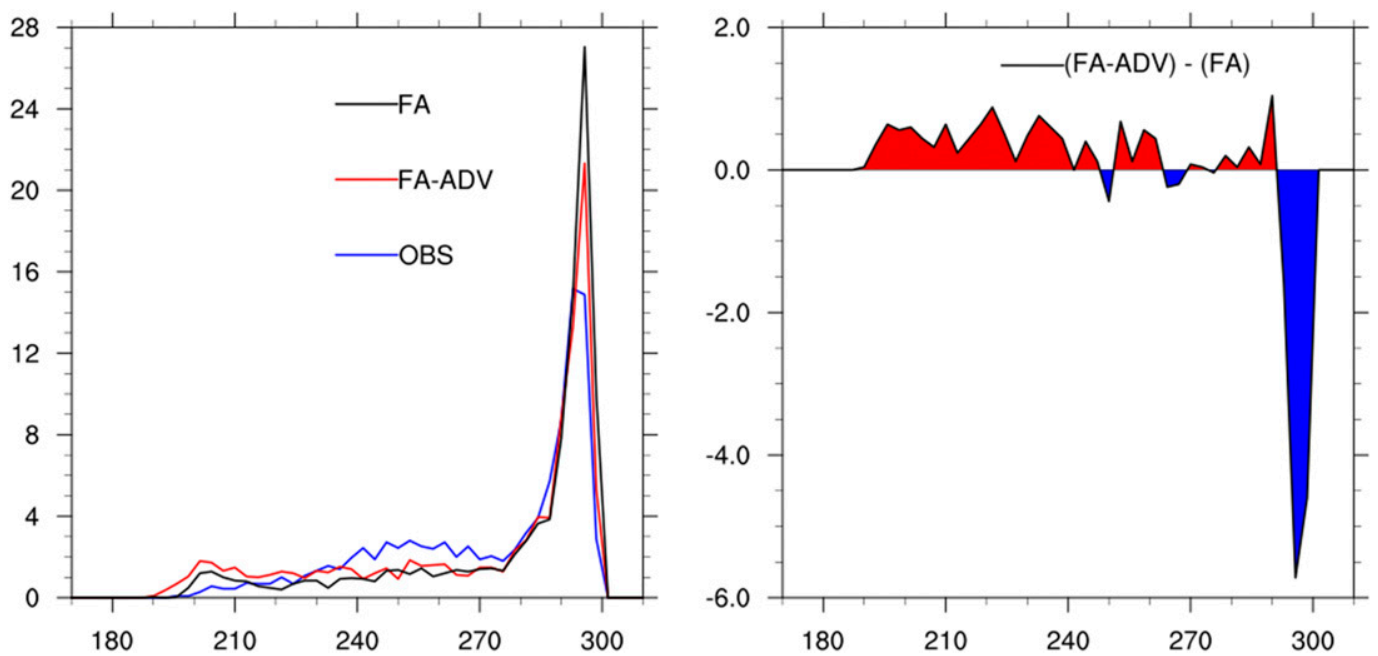

FIG. 3. (left) Probability density function (PDF; \%) of the synthetic FA, FA-adv, and the observed (OBS) IR brightness temperature of Hurricane Matthew at the 96-h forecast in the cycle that started at 0000 UTC 1 Oct 2016. A domain that covers about $2500 \mathrm{~km} \times 2500 \mathrm{~km}$, as shown in Fig. 2, is used for calculation of the PDF. (right) The difference between PDF lines of FA-ADV and FA. 


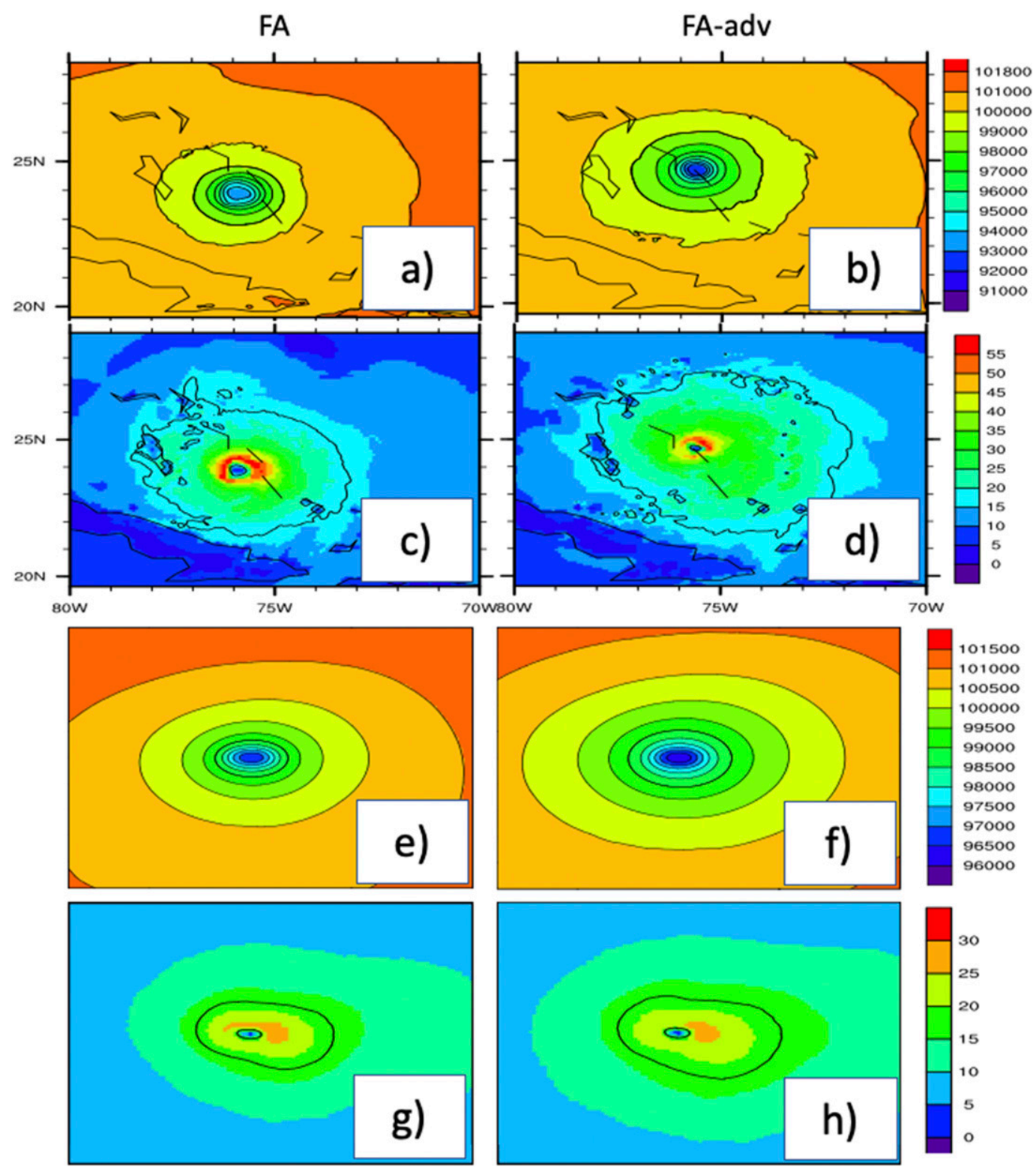

FIG. 4. (a),(b),(e),(f) Sea level pressure (SLP; Pa) and (c),(d),(g),(h) 10-m winds ( $\mathrm{m} \mathrm{s}^{-1}$ ) for (left) FA and (right) FA-adv. Panels (a)-(d) are for a 96-h forecast snapshot of Hurricane Matthew (2016) valid at 0000 UTC 5 Oct 2016, while (e)-(h) are for a composite storm created by averaging 1763 forecast snapshots of Hurricane Matthew. In (c), (d), (g), and (h) the black contour lines show the extension of the area with winds in excess of $34 \mathrm{kt}$.

deeper minimum SLP but weaker 10-m wind speeds, indicating that in FA-adv some points were shifted toward the low pressure low-wind regions in the scatterplot, confirming the same pattern seen in Fig. 4. The mechanisms that caused this characteristic and its implications are discussed later. When compared with the best track $P-V$ plot (Fig. 5, right panel), the results from FA and FA-adv both overestimated the deepening of the SLP with wind speed, as can be seen from the slope of the regression lines.
The simulated tracks and intensities differed significantly between FA and FA-adv. Figure 6 shows the bias of the simulated intensity and the track error of Hurricane Matthew (2016) across 19 cycles. In the early stage of the simulations, with a lead time of $12 \mathrm{~h}$ or less, the FA and FA-adv were nearly identical. This is because in the initial condition the hydrometeor fields were set to zeros, so there was little difference between the advection of separate hydrometeors and the total condensate in the early stage of the simulations. 

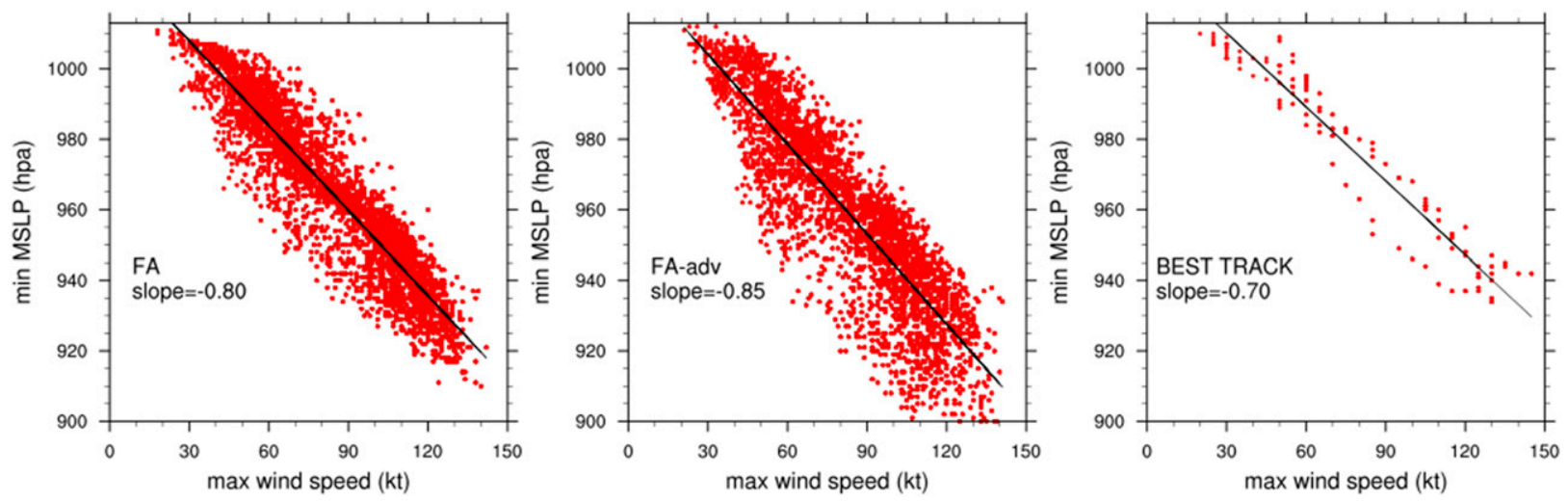

FIG. 5. Pressure-wind scatterplot for FA, FA-adv, and the best track (pressure in hPa and wind speed in kt).

After two days, the minimum SLP from FA-adv became deeper than that in FA, but its maximum 10-m wind was similar or weaker than FA's. This result is consistent with the examples shown in Figs. 4 and 5, highlighting a characteristic that seemingly contradicted the general relationship that a deeper minimum SLP usually means a stronger TC vortex and thus a stronger $10-\mathrm{m}$ wind. The mechanism that caused this discrepancy is discussed in the next section.

\section{Discussion and sensitivity experiments}

To investigate the impact of the different advection methods, namely FA and FA-adv, on the simulated TC size, structure and intensity, it is necessary to review the difference between the implementations of FA and FA-adv. In FA-adv and FA, the same microphysics column subroutine is used. The difference between FA-adv and FA is only in the advection of the hydrometeors, including the rime factor that describes the densities of the precipitation ice to differentiate snow, graupel, and sleet. In FA, before the advection, the hydrometeors are summed to form the total condensate and their fractions are assumed to remain unchanged during the advection. The total condensate is advected using the horizontal and vertical gradients of the total condensate, and after the advection, each hydrometeor mixing ratio is obtained from its respective fraction. After the advection, the new total condensate and the hydrometeor fractions are passed into the microphysics column routine. In FA-adv, the mixing ratios of the different hydrometeors are advected separately. The new mixing ratios after the advection are converted to the total condensate and hydrometeor fractions before being passed to the microphysics column routine. Therefore, this study was focused on the impact of the advection on the hydrometeors as the advection is the only difference in the model code.
In this study, we focus on the vertical advection, considering that any difference in hydrometeor concentrations resulting from the horizontal advection will have less of a dynamical impact on the simulated storm because horizontal advection generally does not cause as significant phase changes or differences in diabatic heating in tropical cyclones as when the vertical motion is involved. The differences in the horizontal advection between FA an FA-adv may also have some secondary impacts on the simulated tropical cyclones which are not discussed in this paper, for example, a broader cloud shield, which would be more likely for situations with upper-level outflow advecting frozen hydrometeors with a small fall speed (like snow), would have radiative impacts, which could in turn impact the kinematic structure of the secondary circulation (Fovell et al. 2016).

Regardless of the numerical method used to implement it, the general formula of the contribution of the vertical advection term to the rate of change of a state variable can be expressed as $-w(\partial F / \partial z)$, where $F$ is the variable that is advected, $w$ is the vertical velocity, and $\partial F / \partial z$ is the vertical gradient of that state variable. A working hypothesis was that if the vertical profiles of Qc and Qr differ significantly, then the total condensate advection scheme in FA could produce different upward advection of Qr and Qc than that in FA-adv. Consequently, the difference in the vertical advection of Qc and Qr between FA and FA-adv could lead to different diabatic heating through latent heat release when the hydrometers undergo phase changes such as condensation and evaporation. Differences in diabatic heating could then cause dynamical differences affecting the storm size and intensity and thus lead to the larger and weaker simulated storms in FA-adv relative to FA that were noted in section 3. This hypothesis is partially inspired by and consistent with Wang (2009), who found that simulated tropical cyclones became larger 

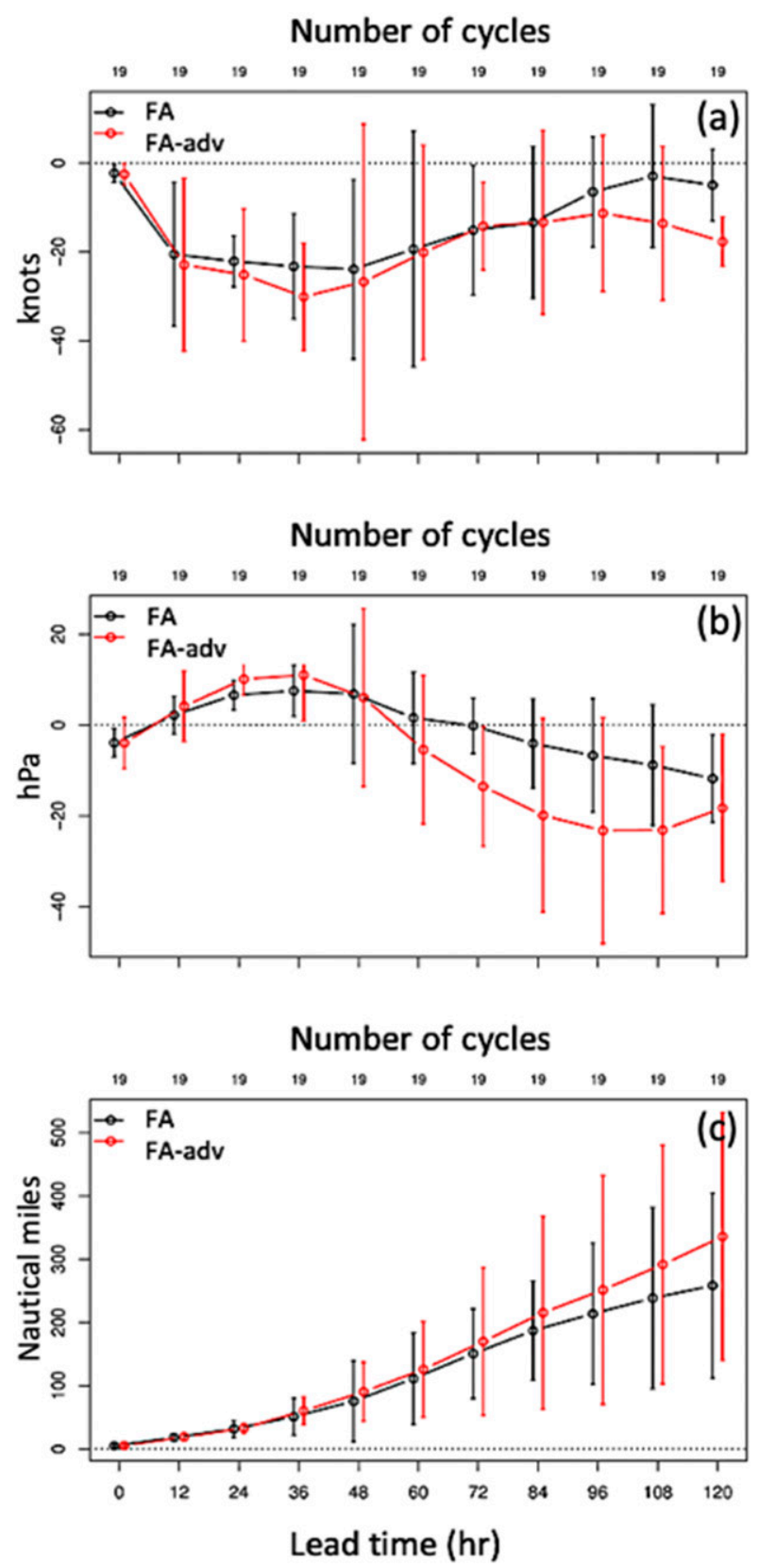

FIG. 6. The (a) 10-m maximum wind bias, (b) minimum SLP bias, and (c) track error for Hurricane Matthew (2016) relative to the best track. Bias is defined as the error averaged over all forecast cycles: bias $=(1 / N) \sum_{1}^{N}$ (forecast - obs) where $N$ is the number of forecast cycles, and National Hurricane Center best tracks are used as observation.

and weaker when artificial warming was introduced in the outer rainbands.

The Hurricane Matthew HWRF simulations were cycled model runs, meaning each cycle used the previous cycle's result as part of its initial condition. The cycling makes it difficult to compare FA and FA-adv, since the runs did not start from identical initial conditions, except for the very first model cycle, when the storm was a weak tropical depression. The real case simulations are also affected nonlinearly by many factors, including environmental wind fields, the vortex initialization procedure, data assimilation, land/ocean coupling, and so on, making it difficult to isolate the effect of the vertical hydrometeor advection scheme. Therefore, in this section, we use the idealized HWRF runs to validate the above hypothesis.

In the first $4 \mathrm{~h}$ of the idealized HWRF simulation, the FA and FA-adv results are nearly identical (not shown), perhaps because the hydrometeors have not developed. Figure 7 shows the difference fields (FA-adv - FA). At $t=5 \mathrm{~h}$, while the Qc from FA was concentrated near $850 \mathrm{hPa}$, the Qc from FA-adv advected farther upward, reaching close to $700 \mathrm{hPa}$ (Fig. 7a). The FA-adv had greater diabatic heating than FA near $850 \mathrm{hPa}$ (Fig. 7d; the mechanism for the diabatic heating distribution will be revisited later in Fig. 9). The greater diabatic heating in FA is accompanied by a stronger updraft (Fig. 7e) and a larger Qr (Fig. 7b) in FA than FA-adv. At $t=5 \mathrm{~h}$, frozen condensate (ice and snow; Qi) has not yet formed so the difference in Qi between FA-adv and FA is close to zero (Fig. 7c). At $t=6 \mathrm{~h}$, it is noticeable that the larger updraft speed and greater diabatic heating in FA-adv than FA have developed further, become stronger, and reached higher levels (Figs. 7i,j). At $t=6 \mathrm{~h}$, Qr (Fig. 7g) and Qi (Fig. 7h) in FA-adv exceeded those in FA and Qc extended much higher in FA-adv than FA (Fig. 7f).

The dramatic 1-h development from the low-level and relatively small extra diabatic heating and updraft in FA-adv than FA (Figs. 7d,e) to those shown in Figs. 7i and $7 \mathrm{j}$, and the associated hydrometeors, can be attributed to a dynamical positive feedback effect triggered by the small difference in diabatic heating at $t=5 \mathrm{~h}$ as shown in Fig. 7d: the greater diabatic heating caused a stronger updraft and thus enhanced the system-scale secondary circulation, which, in turn, intensified the primary circulation (Shapiro and Willoughby 1982). The accelerated primary circulation wind then drove the entropy flux from the ocean surface to the inner core to generate greater diabatic heating from microphysics processes (Rotunno and Emanuel 1987); and the greater diabatic heating led to a stronger updraft and secondary circulation, and so on. This positive dynamical and microphysical feedback effect can be seen from Fig. 8, which shows the breakdown of the heating terms due to the various microphysics processes at $t=6 \mathrm{~h}$. The condensation (pcond) in FA-adv, including that into supercooled Qc water, reached a higher level and released more heat than FA (Figs. 8a,b). The Qi formed mainly by riming of the supercooled Qc (piacwi) and deposition 

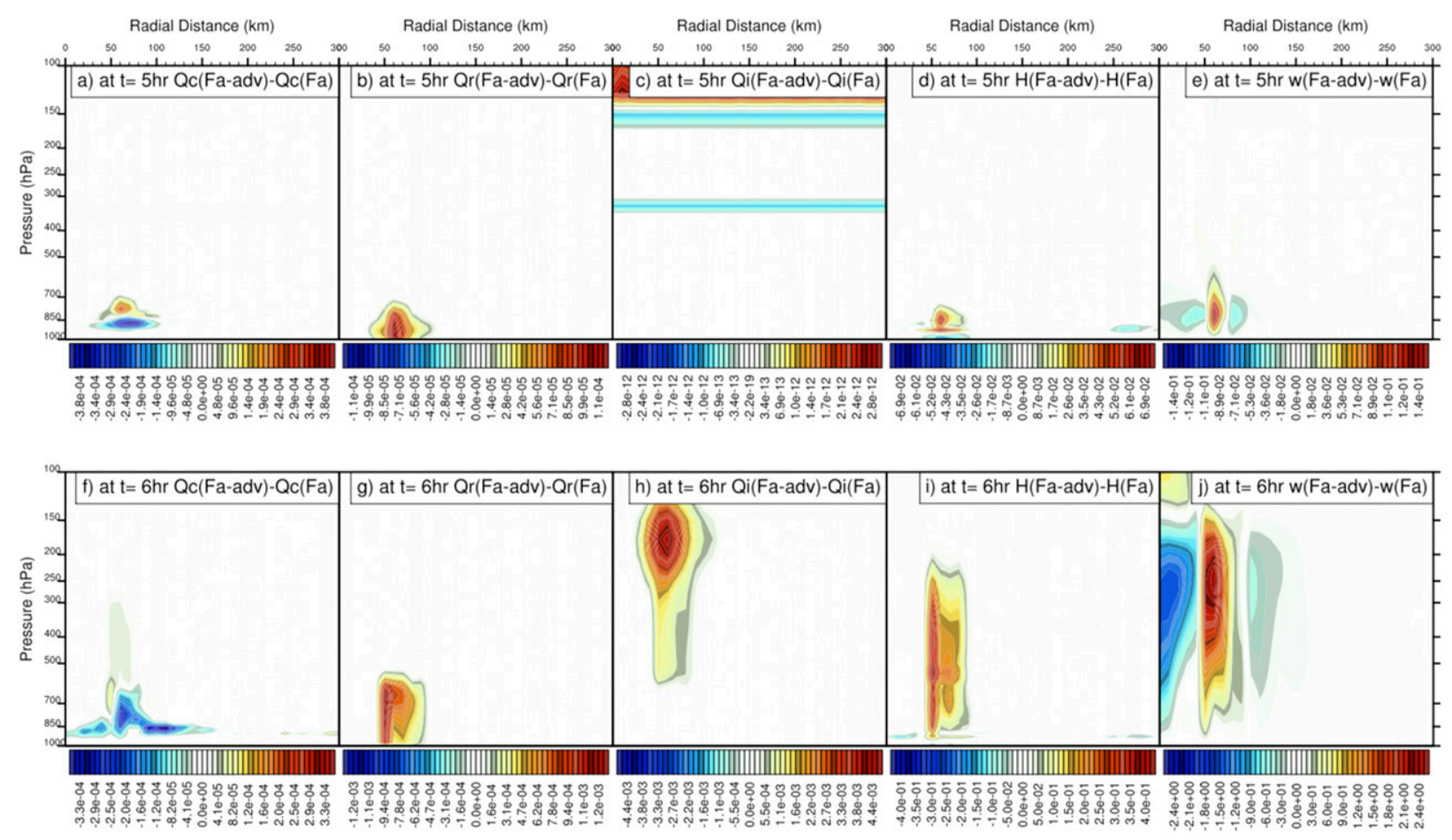

FIG. 7. Idealized HWRF simulation azimuthally averaged results in radial-height coordinates shown for (top) $t=5 \mathrm{~h}$ and (bottom) $t=6 \mathrm{~h}$. (a),(f) Cloud water difference $\left(\mathrm{kg} \mathrm{kg}^{-1}\right),(\mathrm{b}),(\mathrm{g})$ rainwater difference $\left(\mathrm{kg} \mathrm{kg}^{-1}\right)$, (c), (h) frozen condensate difference in ice $+\mathrm{snow}$ $\left(\mathrm{kg} \mathrm{kg}^{-1}\right)$, (d),(i) accumulated diabatic heating difference $(\mathrm{K})$, and (e),(j) difference in vertical velocity $\left(\mathrm{m} \mathrm{s}^{-1}\right)$. All differences are calculated as FA-adv - FA. The $x$ axis is radial distance $(\mathrm{km})$.

(pidep), which were both stronger and thus released more heat in FA-adv (Figs. 8c,e) than FA (Figs. 8d,f). Therefore, the larger Qi in FA-adv than FA (Fig. 7h) is due to the stronger updraft, instead of more low-level Qc being advected upward and turning into Qi.

The initial additional low-level diabatic heating in FA-adv than FA (Fig. 7d), however, was caused by the difference in vertical hydrometeor advection between these two schemes. To further confirm the role of the vertical hydrometeor advection in causing these simulated diabatic heating differences, the advection terms were calculated for $t=7 \mathrm{~h}$ in the idealized HWRF simulation of FA-adv (Fig. 9). From the Qc, Qr, and vertical velocity snapshots, the advection terms were calculated using the following two methods: 1 ) the hydrometeors were advected separately, resembling the method in FA-adv. 2) the hydrometeors were summed to form the total condensate, which was advected; after advection, Qc and Qr were restored using their preadvection fractions, a method similar to FA. These calculations were conducted offline for diagnostic purposes and did not influence the actual simulation.

Figure 9 presents the radius-height plots of the advection of Qc and Qr, showing that the Qc radius-height cross section is separated into two distinct regions by $r=60 \mathrm{~km}$ (Figs. 9a,b). For $r<60 \mathrm{~km}$, there is virtually no vertical gradient of Qc near $850 \mathrm{hPa}$. In Fig. $9, r<60 \mathrm{~km}$ represents the inner core area because upward vertical motion peaked at $60 \mathrm{~km}$ (lines in Figs. 9a-c). For $r>$ $60 \mathrm{~km}$ in Fig. 9, however, there is an evident Qc peak and two vertical gradient extremes, one positive above $850 \mathrm{hPa}$ and the other negative below $850 \mathrm{hPa}$ (Fig. 9d). The separate advection method in FA-adv would result in the advection term shown in Fig. 9g, which shows Qc moving upward between $r=60-90 \mathrm{~km}$ where Qc, the Qc gradient, and Qr coexist (Figs. 9a,b). The Qr (Fig. 9b) is vertically uniform with a slightly higher Qr above $850 \mathrm{hPa}$. The FA-adv method would produce the Qr advection term shown in Fig. 9h. The total condensate Qt is shown in Fig. 9c. In FA, the Qc advection is calculated by partitioning the Qt advection in Fig. 9i by the hydrometeor fractions, which resulted in the Qc advection shown in Fig. 9j and Qr advection shown in Fig. 9k. It can be seen that compared to FA, the FAadv advected more Qc upward above $850 \mathrm{hPa}$ between $r=60-90 \mathrm{~km}$. For $r<60$, the FA-adv had a greater downward advection of Qc than FA, indicating that, after the storm becomes developed, the greater upward advection of Qc in FA-adv occurs mainly in the outer rainbands. In the inner core area, the strong updraft 

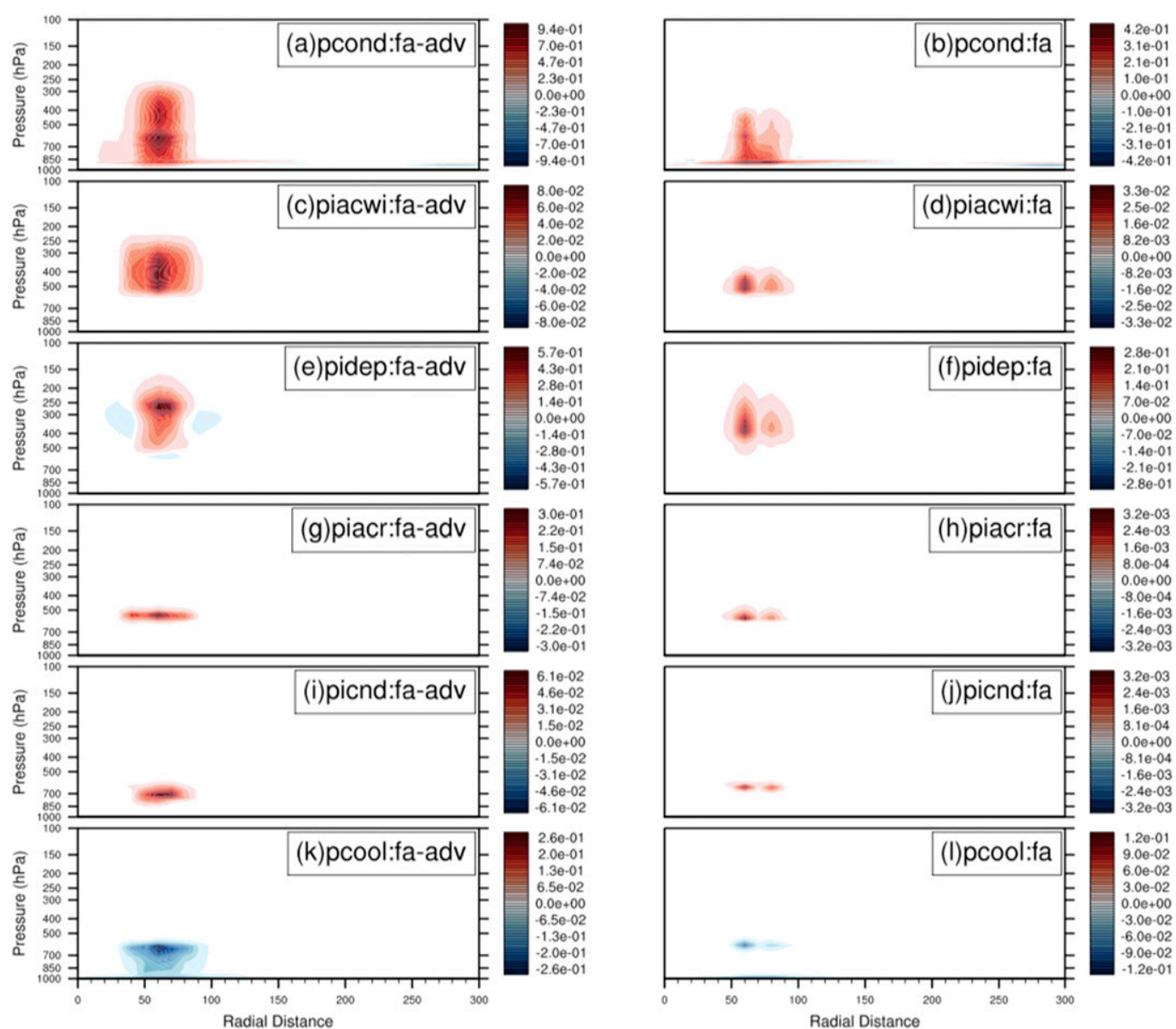

FIG. 8. Vertical cross sections of the idealized HWRF simulation of accumulated temperature change (K), at $t=6 \mathrm{~h}$, as the result of heating or cooling caused by microphysics processes. In the legends, pcond is the condensation $(>0)$ or evaporation $(<0)$, piacwi is the growth of precipitation ice by riming $(>0)$, pidep the deposition $(>0)$ or sublimation $(<0)$ of ice crystals, piacr is the freezing of rain onto large ice at supercooled temperatures $(>0)$, picnd is the condensation onto wet melting ice $(>0)$, and pcool is the sum of three terms (to save plot space) of evaporation from wet melting ice, melting of ice, and rainwater (all three $<0$ ). (left) FA-adv results and (right) FA. Note different color tables are used for different terms as well as for the same term for FA and FA-adv.

causes the Qc profile to be vertically uniform, so that the difference between FA and FA-adv in the inner core area would be small or even opposite in sign relative to the outer rainbands area, as shown in Fig. $9 \mathrm{~g}$ and Fig. 9j. While the FA scheme underestimated the upward Qc advection in the outer rainbands within $r=60-90 \mathrm{~km}$, it overestimated the upward Qr advection in $r=60-90 \mathrm{~km}$, assuming the FA-adv scheme's Qc and Qr vertical advection is the realistic one. Since the vertical gradient of the total condensate (Figs. 9i,1) was much larger than that of the rainwater in the outer rainbands, the rainwater's upward advection was overestimated in FA. If the rainwater had been advected individually, its uniform vertical profile would lead to very little upward advection near the 850-hPa level.
A hypothetical example can help illustrate the overestimation of upward advection of rainwater. For a vertically constant rainwater profile near $850 \mathrm{hPa}$, whose gradient would be zero, the separate advection method in FA-adv would result in a zero upward advection of rainwater; however, the total condensate advection method in FA would produce nonzero upward advection of rainwater because the total condensate gradient was used. In other words, in FA, the large Qc gradients near $850 \mathrm{hPa}$ due to the sharp Qc peak were "shared" by the vertically uniform Qr.

These discrepancies were corrected when the hydrometeors were advected separately in FA-adv, and therefore more Qc was advected upward near $850 \mathrm{hPa}$ in FA-adv than in FA, which can be confirmed by Fig. 7a. Comparing Figs. $7 \mathrm{a}$ and $7 \mathrm{~d}$, it can be seen that the 

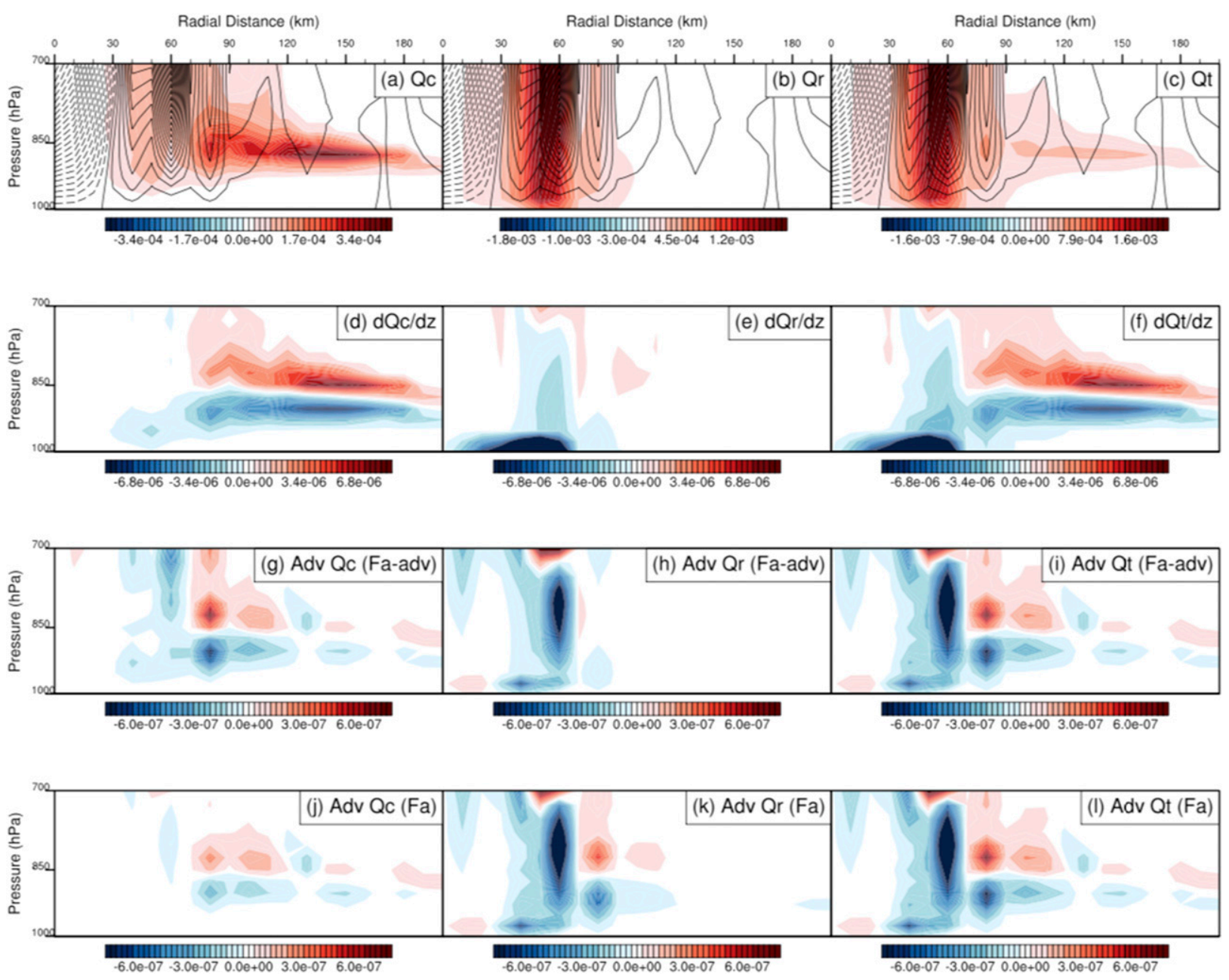

FIG. 9. Advection terms calculated using Qc $\left(\mathrm{kg} \mathrm{kg}^{-1}\right), \mathrm{Qr}\left(\mathrm{kg} \mathrm{kg}^{-1}\right)$, and the vertical velocity $\left(\mathrm{m} \mathrm{s}^{-1}\right)$ in the FA-adv idealized HWRF run results at $t=7 \mathrm{~h}$. (a) Qc, (b) Qr, and (c) the total condensate (Qt; $\mathrm{kg} \mathrm{kg}^{-1}$ ). Vertical velocity is plotted in (a), (b), and (c) as solid lines (updraft) and dashed lines (downdraft). The vertical gradient of (d) Qc $\left(\mathrm{kg} \mathrm{kg}^{-1} \mathrm{~m}^{-1}\right)$, (e) $\mathrm{Qr}\left(\mathrm{kg} \mathrm{kg}^{-1} \mathrm{~m}^{-1}\right)$, and (f) Qt (kg kg$\left.{ }^{-1} \mathrm{~m}^{-1}\right)$. (g) Qc advection $\left(\mathrm{kg} \mathrm{kg}^{-1} \mathrm{~s}^{-1}\right)$, (h) Qr advection $\left(\mathrm{kg} \mathrm{kg} \mathrm{s}^{-1}\right)$, and (i) Qt advection $\left(\mathrm{kg} \mathrm{kg}^{-1} \mathrm{~s}^{-1}\right)$ using FA-adv. (j)-(l) The advection terms using FA. The $x$ axis is the radial distance $(\mathrm{km})$.

greater initial diabatic heating in FA-adv (before the dynamic positive feedback effect takes over) is a local effect that may be attributed to the more upward Qc advection in FA-adv. In summary, Figs. 7-9 show that the difference in the vertical hydrometeor advection methods between the FA and FA-adv affects the advection of hydrometeors and the associated temperature field and dynamical processes, and the effects are different for different radial areas. For the outer rainbands area near $r=60-90 \mathrm{~km}$, the peaked structure of Qc and the vertically uniform Qr coexist (Figs. 9a,b), which is sufficient for the differences in vertical advection in the FA and FA-adv schemes to take effect. In the inner core $(r<60 \mathrm{~km})$, the strong updraft helps reduce the Qc peak and makes Qc vertically uniform, similar to $Q r$, as shown in Fig. 9a, so the difference between the FA and FA-adv simulated advection is small or opposite in sign to that in the outer rainbands (Figs. 9g,j).

The artificial difference in diabatic heating between FA and FA-adv has a thermodynamic effect on hurricane size and intensity. Figure 10 showed the azimuthally averaged pressure-radial cross sections of hydrometeors (Figs. 10a-f), vertical velocity (Figs. 10g,h) and tangential wind (Figs. 10i,j) of a mature Hurricane Matthew (2016) simulated by HWRF using FA (left) and FA-adv (right). The greater diabatic heating in FAadv simulated larger-sized storm. The cloud, rain, and frozen condensate in FA extended radially to 200, 250, and $300 \mathrm{~km}$; but they reached farther to 300,350 , and $400 \mathrm{~km}$, respectively, in FA-adv. The updraft and tangential wind areas in FA-adv also extended farther than FA. The larger storm simulated by FA-adv was also 

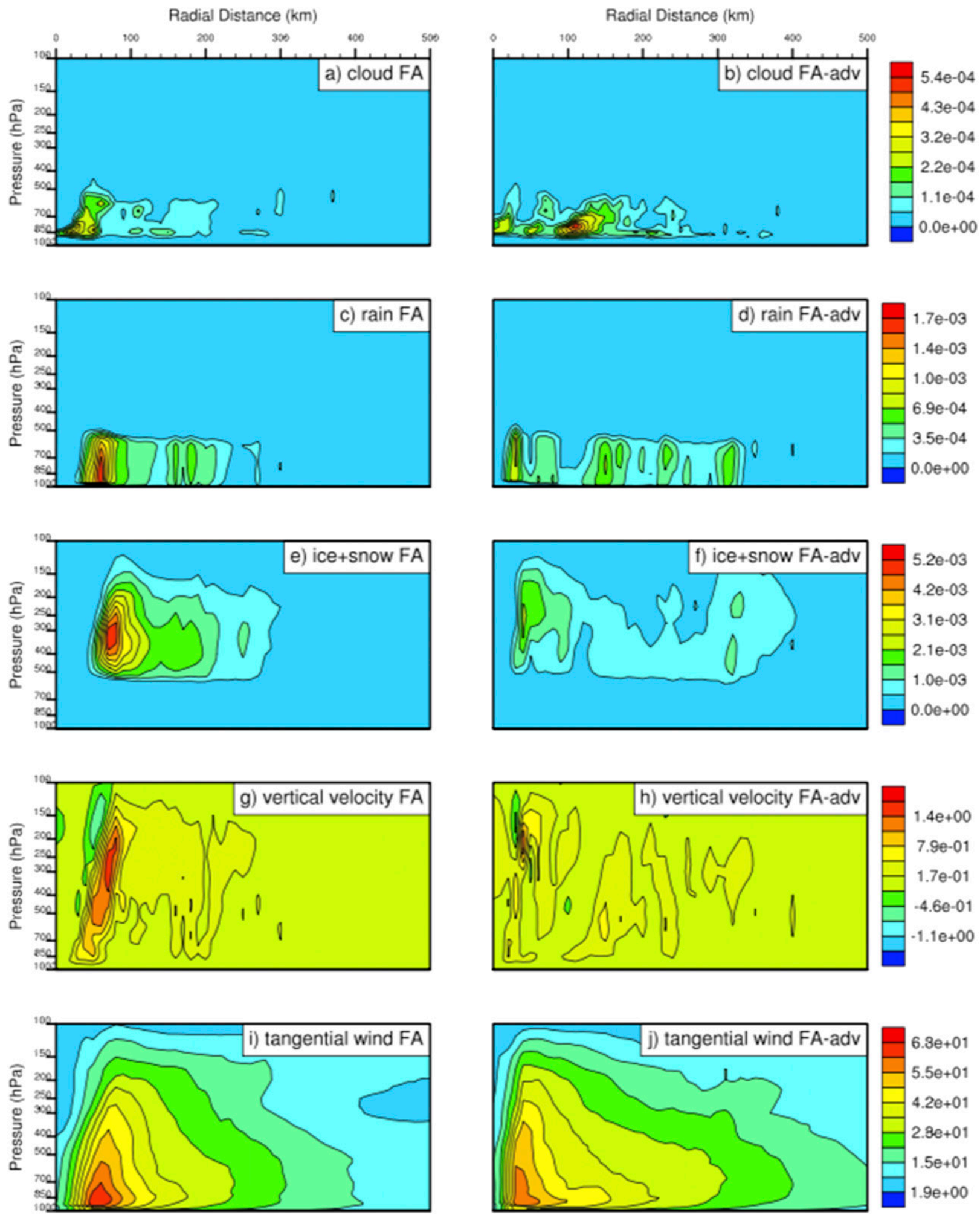

FIG. 10. Azimuthally averaged pressure-radial cross sections of (a),(b) Qc $\left(\mathrm{kg} \mathrm{kg}^{-1}\right)$, (c), (d) $\mathrm{Qr}\left(\mathrm{kg} \mathrm{kg}^{-1}\right)$, (e),(f) Qi + Qs $\left(\mathrm{kg} \mathrm{kg}^{-1}\right)$, (g),(h) vertical velocity $\left(\mathrm{m} \mathrm{s}^{-1}\right)$, and (i),(j) tangential wind $\left(\mathrm{m} \mathrm{s}^{-1}\right)$ of Hurricane Matthew (2016) simulated by HWRF using (left) FA and (right) FA-adv. The simulations were initialized at 0000 UTC 1 Oct 2016 with a lead time of $96 \mathrm{~h}$. 
more powerful by the integrated kinetic energy (IKE), defined as $\int_{0}^{V}(1 / 2) \rho U^{2} d v$, where $V$ is the volume of the domain covering a radial distance of $500 \mathrm{~km}$ and in the vertical from $100 \mathrm{hPa}$ to the surface, $\rho$ is air density which is a function of altitude, and $U$ is tangential wind (Powell and Reinhold 2007). The IKE in Fig. 10i was $7.3 \times 10^{4}$ terajoules (TJ) and in Fig. 10j, it was $8.8 \times 10^{4}$ TJ. Although the FA-adv simulated storm is larger and more powerful, its peak intensity is weaker than FA. As illustrated in Fig. 9, the artificial difference in diabatic heating between FA and FA-adv occurs where both Qc and Qr exist. From Figs. 10a-d it is clear that most of the areas where Qc and Qr coexist are outside of eyewall. Wang (2009) showed the net diabatic heating outside the eyewall causes pressure to fall in the lower troposphere and reduce the pressure gradient across the eyewall, which weakens the tangential wind near RMW but increase the size the TC vortex. The findings in this paper are similar to those in Wang (2009), who found that artificial diabatic heating (cooling) added to the outer rainbands at $60 \mathrm{~km}<r<90 \mathrm{~km}$ causes the intensity of the storm to weaken (strengthen) and the storm size to enlarge (shrink). While Wang (2009) artificially added heating or cooling to model experiments, this paper identified the same effect from heating to weaken and enlarge the storms, but the heating described in this paper results from the different hydrometeor advection schemes in FA-adv and FA, instead of being artificially added as in Wang (2009).

\section{Conclusions}

The advection of the total condensate in the FA scheme underestimates the upward advection of Qc and overestimates the upward advection of low-level Qr. The separate advection of hydrometeors in the FA-adv scheme, in theory, corrected this problem, which causes the difference between these two schemes in their simulated TC microphysics fields and dynamics. The differences mainly originated from the greater upward advection of Qc in FA-adv than in FA. The greater vertical advection of Qc in FA-adv represents a continual source of cloud water freezing into cloud ice. The additional diabatic heating from this phase change in FA-adv affects the dynamics of the simulated TC through hydrostatic adjustment. The radial distribution of the azimuthally averaged extra diabatic heating in FA-adv causes an SLP adjustment that, according to the cyclostrophic balance and gradient wind balance relations, leads to a weaker maximum wind speed but stronger wind speed farther away from the storm inner core, and causes the storm's size to expand. Therefore, although the integrated kinetic energy is larger in FA-adv, its maximum 10-m wind speed is weaker than in FA.

It should be emphasized that the root of the differences in results between FA and FA-adv is the characteristics of the vertical profile of Qc, which has a sharp gradient near $850 \mathrm{hPa}$ in the outer rainbands, and that of $\mathrm{Qr}$, which is vertically uniform. In FA, the resultant total condensate profile has a gradient near $850 \mathrm{hPa}$ that is smaller than Qc but larger than Qr. When the total condensate is advected and partitioned back to cloud water and Qr, the advection of Qc is underestimated and that of Qr is overestimated.

Although in theory the separate advection of hydrometeor species in FA-adv is more realistic than the advection of total condensate in FA, this evaluation showed that FA-adv simulated much larger storms than FA, and therefore degraded the HWRF performance. One motivation for testing the FA-adv scheme in HWRF was an earlier finding showing that the Thompson et al. (2008) scheme, with its inherent separate advection of hydrometeor species, produced a degradation of TC track and intensity in the east Pacific (although an improvement was noted in the Atlantic basin; Biswas et al. 2016). Given that it is not possible to run the Thompson scheme without separate species advection, we used a scheme that allows both total and separate advection to investigate whether the advection by itself could cause the degradation. It should be noted that HWRF, like other NWP models, is a very complex nonlinear system, and tuning in other parts of the model system could have masked errors introduced by the total condensate advection ( $\mathrm{Bu}$ et al. 2017). That is why in this study we focused on understanding the mechanisms that are responsible for the simulated discrepancies between FA and FA-adv, instead of seeking to improve the HWRF performance with FAadv. Future work on examining the related physics schemes, such as radiation, cumulus convection, or PBL, is needed to identify those tunings so that the separate hydrometeor advection can achieve better forecast performance.

Acknowledgments. Shaowu Bao received support for this project through the Developmental Testbed Center (DTC) Visitor Program. The DTC is funded by the National Oceanic and Atmospheric Administration, the Air Force, the National Center for Atmospheric Research, and the National Science Foundation. The National Center for Atmospheric Research is sponsored by the National Science Foundation. Evan Kalina is supported by funding from NOAA Award NA17OAR4320101. The computation has been conducted using resources from the NOAA Research and 
Development High Performance Computing Program (URL:http://rdhpcs.noaa.gov) and the Major Research Instrumentation program at the National Science Foundation under Award AGS-1624068. The three anonymous reviewers provided many insightful and helpful suggestions and comments, which are greatly appreciated. We also thank Drs. Ferrier, Aligo, Abarca, and Liu at NOAA's Environmental Modeling Center (EMC) and Dr. Fovell at the University of Albany, for their insightful comments and suggestions that helped the authors improve the manuscript.

\section{REFERENCES}

Aligo, E., B. Ferrier, J. Carley, E. Rogers, M. Pyle, S. J. Weiss, and I. L. Jirak, 2014: Modified microphysics for use in highresolution NAM forecasts. 27th Conf. on Severe Local Storms, Madison, WI, Amer. Meteor. Soc., 16A.1, https://ams.confex.com/ ams/27SLS/webprogram/Paper255732.html.

,-- , and J. R. Carley, 2018: Modified NAM microphysics for forecasts of deep convective storms. Mon. Wea. Rev., 146, 4115-4153, https://doi.org/10.1175/MWR-D-17-0277.1.

Bao, J.-W., S. G. Gopalakrishnan, S. A. Michelson, F. D. Marks, and M. T. Montgomery, 2012: Impact of physics representations in the HWRFX on simulated hurricane structure and pressure-wind relationships. Mon. Wea. Rev., 140, 3278-3299, https://doi.org/10.1175/MWR-D-11-00332.1.

Biswas, M., C. Holt, G. Thompson, L. Bernardet, and K. Newman, 2016: Testing Thompson microphysics with Hurricane WRF model. Developmental Testbed Center, 31 pp., https://dtcenter.org/eval/hwrf_thomp2016/Thompson_HWRF_ Report_July2016.pdf.

—, L. Carson, K. Newman, L. Bernardet, E. Kalina, E. Grell, and J. Frimel, 2017: Community HWRF users guide v3.9a. NOAA Tech. Memo. OAR GSD-51, 153 pp., https://doi.org/10.7289/ V5/TM-OAR-GSD-51.

_ Forecasting (HWRF) Model: 2017 scientific documentation. NCAR Tech. Note NCAR/TN-544+STR, 111 pp., https:// doi.org/10.5065/D6MK6BPR.

Bu, Y. P., R. G. Fovell, and K. L. Corbosiero, 2017: The influences of boundary layer mixing and cloud-radiative forcing on tropical cyclone size. J. Atmos. Sci., 74, 1273-1292, https:// doi.org/10.1175/JAS-D-16-0231.1.

Carrasco, C. A., C. W. Landsea, and Y.-L. Lin, 2014: The influence of tropical cyclone size on its intensification. Wea. Forecasting, 29, 582-590, https://doi.org/10.1175/WAF-D-13-00092.1.

Cintineo, R., J. A. Otkin, F. Kong, and M. Xue, 2014: Evaluating the erformance of planetary boundary layer and cloud microphysical parameterization schemes in convection-permitting ensemble forecasts using synthetic GOES-13 satellite observations. Mon. Wea. Rev., 142, 163-182, https://doi.org/10.1175/MWR-D13-00143.1.

Eikenberg, S., C. Köhler, A. Seifert, and S. Crewell, 2015: How microphysical choices affect simulated infrared brightness temperatures. Atmos. Res., 156, 67-79, https://doi.org/10.1016/ j.atmosres.2014.12.010.

Ferrier, B. S., 1994: A double-moment multiple-phase fourclass bulk ice scheme. Part I: Description. J. Atmos. Sci., 51, 249-280, https://doi.org/10.1175/1520-0469(1994)051<0249: ADMMPF $>2.0 . \mathrm{CO} ; 2$.
— Y. Y. Jin, Y. Lin, T. Black, E. Rogers, and G. DiMego, 2002: Implementation of a new grid-scale cloud and precipitation scheme in the NCEP Eta model. Preprints, 15th Conf. on Numerical Weather Prediction, San Antonio, TX, Amer. Meteor. Soc., 280-283.

Fovell, R. G., and H. Su, 2007: Impact of cloud microphysics on hurricane track forecasts. Geophys. Res. Lett., 34, L24810, https://doi.org/10.1029/2007GL031723.

— Y. P. Bu, K. L. Corbosiero, W. W. Tung, Y. Cao, H. C. Kuo, L. H. Hsu, and H. Su, 2016: Multiscale convection-coupled systems in the tropics. Influence of Cloud Microphysics and Radiation on Tropical Cyclone Structure and Motion, Meteor. Monogr., No. 56, Amer. Meteor. Soc., https://doi.org/10.1175/ AMSMONOGRAPHS-D-15-0006.1.

Gopalakrishnan, S. G., F. Marks, X. Zhang, J.-W. Bao, K.-S. Yeh, and R. Atlas, 2011: The experimental HWRF system: A study on the influence of horizontal resolution on the structure and intensity changes in tropical cyclones using an idealized framework. Mon. Wea. Rev., 139, 1762-1784, https://doi.org/ 10.1175/2010MWR3535.1.

_,- J. J. Zhang, X. Zhang, J.-W. Bao, and V. Tallapragada, 2013: A study of the impacts of vertical diffusion on the structure and intensity of the tropical cyclones using the highresolution HWRF system. J. Atmos. Sci., 70, 524-541, https:// doi.org/10.1175/JAS-D-11-0340.1.

Janjić, Z. I., 2003: A nonhydrostatic model based on a new approach. Meteor. Atmos. Phys., 82, 271-285, https://doi.org/ 10.1007/s00703-001-0587-6.

Lin, Y.-L., R. D. Farley, and H. D. Orville, 1983: Bulk parameterization of the snow field in a cloud model. J. Climate Appl. Meteor., 22, 1065-1092, https://doi.org/10.1175/15200450(1983)022<1065:BPOTSF $>2.0 . \mathrm{CO} ; 2$.

Morrison, H., and J. A. Milbrandt, 2015: Parameterization of cloud microphysics based on the prediction of bulk ice particle properties. Part I: Scheme description and idealized tests. J. Atmos. Sci., 72, 287-311, https://doi.org/10.1175/JASD-14-0065.1.

Otkin, J. A., W. E. Lewis, A. J. Lenzen, B. D. McNoldy, and S. J. Majumdar, 2017: Assessing the accuracy of the cloud and water vapor fields in the Hurricane WRF (HWRF) model using satellite infrared brightness temperatures. Mon. Wea. Rev., 145, 2027-2046, https://doi.org/10.1175/ MWR-D-16-0354.1.

Pattnaik, S., and T. N. Krishnamurti, 2007: Impact of cloud microphysical processes on hurricane intensity. Part 2: Sensitivity experiments. Meteor. Atmos. Phys., 97, 127-147, https:// doi.org/10.1007/s00703-006-0248-x.

Powell, M. D., and T. A. Reinhold, 2007: Tropical cyclone destructive potential by integrated kinetic energy. Bull. Amer. Meteor. Soc., 88, 513-526, https://doi.org/10.1175/BAMS-88-4-513.

Rogers, E., T. Black, B. Ferrier, Y. Lin, D. Parrish, and G. DiMego, 2001: Changes to the NCEP Meso Eta Analysis and Forecast System: Increase in resolution, new cloud microphysics, modified precipitation assimilation, modified 3DVAR analysis. NWS Tech. Procedures Bull., 488 pp., http://www.emc.ncep.noaa.gov/mmb/mmbpll/eta12tpb.

_ and Coauthors, 2009: The NCEP North American mesoscale modeling system: Recent changes and future plans. $23 \mathrm{rd}$ Conf. on Weather Analysis and Forecasting/19th Conf. on Numerical Weather Prediction, Omaha, NE, Amer. Meteor. Soc., 2A.4, http://ams.confex.com/ams/pdfpapers/154114.pdf.

Rotunno, R., and K. A. Emanuel, 1987: An air-sea interaction theory for tropical cyclones. Part II: Evolutionary study using a 
nonhydrostatic axisymmetric numerical model. J. Atmos. Sci., 44, 542-561, https://doi.org/10.1175/1520-0469(1987)044<0542: AAITFT $>2.0 . \mathrm{CO} ; 2$.

Shapiro, L. J., and H. E. Willoughby, 1982: The response of balanced hurricanes to local sources of heat and momentum. J. Atmos. Sci., 39, 378-394, https://doi.org/10.1175/15200469(1982)039<0378:TROBHT>2.0.CO;2.

Sobhani, N., and D. D. Vento, 2017: Performance analysis and optimization of the Weather Research and Forecasting Model (WRF) advection schemes. Third Symp. on High Performance Computing for Weather, Water, and Climate, Seattle, WA, Amer. Meteor. Soc., 3.4, https://ams.confex.com/ams/ 97Annual/webprogram/Paper315832.html.

Thompson, G., and T. Eidhammer, 2014: A study of aerosol impacts on clouds and precipitation development in a large winter cyclone. J. Atmos. Sci., 71, 3636-3658, https://doi.org/ 10.1175/JAS-D-13-0305.1.

, P. R. Field, R. M. Rasmussen, and W. D. Hall, 2008: Explicit forecasts of winter precipitation using an improved bulk microphysics scheme. Part II: Implementation of a new snow parameterization. Mon. Wea. Rev., 136, 5095-5115, https://doi.org/10.1175/2008MWR2387.1.

- M. Tewari, K. Ikeda, S. Tessendorf, C. Weeks, J. A. Otkin, and F. Kong, 2016: Explicitly-coupled cloud physics and radiation parameterizations and subsequent evaluation in WRF high-resolution convective forecasts. Atmos. Res., 168, 92-104, https://doi.org/10.1016/j.atmosres.2015.09.005.

Wang, Y., 2009: How do outer spiral rainbands affect tropical cyclone structure and intensity? J. Atmos. Sci., 66, 1250-1273, https://doi.org/10.1175/2008JAS2737.1.

Zhang, J., and F. Marks, 2014: Sensitivity of hurricane intensity and structure to horizontal diffusion in idealized HWRF simulations. 2014 Fall Meeting, San Francisco, CA, Amer. Geophys. Union, Abstract A12F-07.

Zhu, T., and D.-L. Zhang, 2006: Numerical simulation of Hurricane Bonnie (1998). Part II: Sensitivity to varying cloud microphysical processes. J. Atmos. Sci., 63, 109-126, https://doi.org/ 10.1175/JAS3599.1. 